\title{
Effect of autologous platelet-rich plasma on the chondrogenic differentiation of rabbit adipose-derived stem cells in vitro
}

\author{
XIAO-BO TANG ${ }^{1 *}$, PEI-LONG DONG ${ }^{1 *}$, JIAN WANG $^{1}$, HAI-YANG ZHOU ${ }^{2}$, \\ HAI-XIANG ZHANG ${ }^{3}$ and SHAN-ZHENG WANG ${ }^{3}$ \\ ${ }^{1}$ Department of Orthopedics, Jianhu Hospital, Medical School of Nantong University, Jianhu, Jiangsu 224700; \\ ${ }^{2}$ Department of Orthopedics, Nanjing Integrative Medicine Hospital, Nanjing University of Traditional Chinese Medicine, \\ Nanjing, Jiangsu 210014; ${ }^{3}$ Department of Orthopedics, Zhongda Hospital, Medical School of Southeast University, \\ Nanjing, Jiangsu 210009, P.R. China
}

Received June 12, 2014; Accepted January 16, 2015

DOI: $10.3892 / \mathrm{etm} .2015 .2528$

\begin{abstract}
This study aimed to isolate rabbit adipose-derived stem cells (ADSCs) and explore the potential of platelet-rich plasma (PRP) in the chondrogenic differentiation of ADSCs, thereby potentially providing a new approach for the repair and regeneration of cartilage injury. Rabbit ADSCs were isolated and characterized by induction towards adipogenic, osteogenic and chondrogenic lineages in vitro. The isolated ADSCs were also cultured with or without $10 \%$ PRP. Immunofluorescence staining, toluidine blue staining and reverse transcription-quantitative polymerase chain reaction (RT-qPCR) were used to detect type II collagen (Col II) and aggrecan (AGC) expression. Col II immunofluorescence staining and toluidine blue staining indicated that following induction by autologous PRP, ADSCs manifested Col II and AGC expression. The expression of Col II and AGC mRNA was significantly upregulated in the PRP-treated cells when compared with that in control cells. Autologous PRP produced by laboratory centrifugation was able to promote the chondrogenic differentiation of rabbit ADSCs in vitro.
\end{abstract}

\section{Introduction}

Adult stem cells are a class of undifferentiated cells, exhibiting long-term self-renewal proliferative and multi-lineage differentiation potential (1). These cells can be obtained from multiple types of tissue, including bone marrow, tendon and

Correspondence to: Dr Shan-Zheng Wang, Department of Orthopedics, Zhongda Hospital, Medical School of Southeast University, 87 Ding Jia Qiao Road, Nanjing, Jiangsu 210009, P.R. China

E-mail: wang_seu_edu@163.com

*Contributed equally

Key words: adipose-derived stem cells, platelet-rich plasma, chondrogenic differentiation, growth factors adipose. Due to these characteristics, the application of such cells for tissue repair and regeneration has attracted the attention of many researchers. As a member of the adult stem cell family, adipose-derived stem cells (ADSCs) are accessible and possess strong self-renewal capacity; thus, they may serve as the ideal seeding cells for tissue regeneration (2).

Platelet-rich plasma (PRP) is a fraction of the whole blood, with the platelet concentration above the baseline level (3). PRP can be extracted by the centrifugation of whole blood and contains a high concentration of platelets, fibrin and white blood cells. The concept that PRP could promote tissue regeneration and cell differentiation is based on the role of platelets $(4,5)$. When activated, a variety of growth factors, including transforming growth factor- $\beta$ (TGF- $\beta$ ), platelet derived growth factor (PDGF), insulin-like growth factor-1 (IGF-1), vascular endothelial growth factor (VEGF), basic fibroblastic growth factor (bFGF) and epidermal growth factor (EGF) are secreted by the platelets (5-7). The growth factors present in PRP play a critical role in tissue repair and regeneration (8).

The repair of articular cartilage defects has long been an obstacle for orthopedic studies $(9,10)$. Tissue engineering is a promising option for cartilage regeneration $(11,12)$ when an appropriate class of seeding cells is selected, particularly adult stem cells with the advantage of strong proliferative and differentiative activity. Thus, ADSCs as an accessible source of seeding cells for cartilage regeneration, when induced towards the chondrogenic lineage, are a promising prospect for the creation of tissue-engineered cartilage.

The present study primarily isolated and characterized ADSCs from rabbits, and then explored the ability of PRP obtained by laboratory centrifugation to induce ADSCs towards the chondrogenic lineage, with the aim of providing a new approach for cartilage regeneration.

\section{Materials and methods}

Animal treatment. All experimental procedures involving animals conformed with the National Institutes of Health (NIH) Guide for the Care and Use of Laboratory Animals and were approved by the Administration Committee of Experimental Animals, Jiangsu, China. 
Isolation and culture of ADSCs. Ten 4-month-old New Zealand white rabbits (2.8-3.5 kg; Jiangsu Academy of Agricultural Sciences, Nanjing, China) were used. Under sterile conditions, adipose tissues $(\sim 10 \mathrm{~g})$ were carefully taken from the rabbits following anesthesia. Anesthesia was induced via an intravenous injection of ketamine hydrochloride $(60 \mathrm{mg} / \mathrm{kg})$ and xylazine $(6 \mathrm{mg} / \mathrm{kg}$ ) (Shanghai Tongwei Biological Technology Co., Ltd., Shanghai, China). These samples were minced and then digested for $1 \mathrm{~h}$ at $37^{\circ} \mathrm{C}$ with type I collagenase (2.5 mg/ml; Sigma-Aldrich, St Louis, MO, USA). During the digestion time, these tissues were swiftly shaken every $20 \mathrm{~min}$. The digested samples were passed through a $70-\mu \mathrm{m}$ cell strainer (Becton Dickinson, Franklin Lakes, NJ, USA) to yield a single-cell suspension. These released cells were washed with phosphate-buffered saline (PBS) and re-suspended in complete culture medium containing low-glucose Dulbecco's modified Eagle's medium (LG-DMEM; Gibco Life Technologies, Carlsbad, CA, USA), $10 \%$ fetal bovine serum (FBS), $100 \mathrm{U} / \mathrm{ml}$ penicillin, $100 \mu \mathrm{g} / \mathrm{ml}$ streptomycin and $2 \mathrm{mM}$ L-glutamine (all from Invitrogen Life Technologies, Carlsbad, CA, USA) at a density of 500 cells $/ \mathrm{cm}^{2}$. The resuspended cells were cultured in a humidified atmosphere containing $5 \% \mathrm{CO}_{2}$ at $37^{\circ} \mathrm{C}$. At day 3 after initial plating, the cells were washed twice with PBS to remove the non-adherent cells. At day seven to ten, these colonies were trypsinized and mixed together as passage 0 .

Multidifferentiation potential. The osteogenic, adipogenic and chondrogenic differentiation potential of adipose-derived stem cells at passage 3 was investigated according to the method of Rui et al (13) with certain modifications, as described below.

Adipogenic differentiation assays. ADSCs were plated at $4 \times 10^{3}$ cells $/ \mathrm{cm}^{2}$ in a six-well plate and cultured in complete culture medium until the cells reached confluence. The medium was replaced with complete medium or adipogenic medium, which was complete culture medium and adipogenic reagents. The adipogenic reagents included dexamethasone $(500 \mathrm{nM})$, isobutylmethylxanthine $(0.5 \mathrm{mM})$, indomethacin $(50 \mathrm{mM})$ and insulin $(10 \mathrm{mg} / \mathrm{ml})$ (all from Sigma-Aldrich). The culture medium was replaced every 3-4 days. After 21 days of incubation, Oil Red O staining was performed to confirm the formation of oil droplets. For Oil Red O staining, the cells were fixed in $70 \%$ ethanol for $10 \mathrm{~min}$ and stained with $0.3 \%$ fresh Oil Red O solution (Sigma-Aldrich) for $2 \mathrm{~h}$.

Osteogenic differentiation assays. ADSCs were plated at the same density as in the previously described adipogenic assays. Then, these cells were cultured with complete medium or osteogenic medium, which were complete culture medium and osteogenic reagents. The osteogenic reagents included dexamethasone (1 $\mathrm{nM})$, ascorbic acid $(50 \mathrm{mM})$ and $\beta$-glycerolphosphate ( $20 \mathrm{mM}$ ) (all from Sigma-Aldrich). The culture medium was replaced every 3-4 days. After 28 days of incubation, $0.5 \%$ alizarin red ( $\mathrm{pH} 4.1$; Sigma-Aldrich) was utilized to stain the cells for $30 \mathrm{~min}$, subsequent to the cells being fixed in $70 \%$ ethanol for $10 \mathrm{~min}$.

Chondrogenic differentiation assays. A pellet culture system was used for chondrogenic differentiation. Approximately
$8 \times 10^{5}$ ADSCs were centrifuged at $450 \times \mathrm{g}$ for $10 \mathrm{~min}$ in a $15-\mathrm{ml}$ conical polypropylene tube to form a micromass and incubated in complete medium or a chondrogenic medium that comprised complete culture medium and chondrogenic reagents. The chondrogenic reagents comprised LG-DMEM, supplemented with $10 \mathrm{ng} / \mathrm{ml}$ TGF- $\beta 3$ (R\&D Systems, Minneapolis, MN, USA), $500 \mathrm{ng} / \mathrm{ml}$ bone morphogenetic protein-2 (BMP-2; R\&D Systems), $10^{-7} \mathrm{M}$ dexamethasone, $50 \mu \mathrm{g} / \mathrm{ml}$ ascorbate-2-phosphate, $40 \mu \mathrm{g} / \mathrm{ml}$ proline, $100 \mu \mathrm{g} / \mathrm{ml}$ pyruvate (all from Sigma-Aldrich) and 1:100 diluted ITS $^{+}$ Premix [6.25 mg/ml insulin, $6.25 \mathrm{mg} / \mathrm{ml}$ transferrin, $6.25 \mathrm{mg} / \mathrm{ml}$ selenous acid, $1.25 \mathrm{mg} / \mathrm{ml}$ bovine serum albumin (BSA) and $5.35 \mathrm{mg} / \mathrm{ml}$ linoleic acid; Becton Dickinson]. After 21 days of incubation, the pellet was fixed for histology and the immunohistochemical staining of collagen type II (Col II).

Immunohistochemical staining of Col II. The immunohistochemical staining was performed as follows. Briefly, paraffin-embedded sections were deparaffinized in xylene and dehydrated through a graded series of alcohol. Then, dewaxed slices of cell mass were incubated in $3 \%$ hydrogen peroxide at room temperature for $20 \mathrm{~min}$. Antigen retrieval was performed with $2 \mathrm{mg} / \mathrm{ml}$ protease (Calbiochem, Bie and Berntsen, Rødovre, Denmark) at $37^{\circ} \mathrm{C}$ for $30 \mathrm{~min}$ for $\mathrm{Col}$ II detection. The sections were incubated with mouse monoclonal antibody against rabbit Col II (cat. no. sc-52658, Santa Cruz Biotechnology, Inc., Dallas, TX, USA; 1:100 dilution with 5\% goat serum in PBS containing $1 \% \mathrm{BSA}$ ) overnight at $4^{\circ} \mathrm{C}$ after blocking with $5 \%$ goat serum for $20 \mathrm{~min}$ at room temperature. The spatial localization of Col II was observed by incubating with goat polyclonal anti-mouse IgG tetramethylrhodamine-conjugated secondary antibody (cat. no T5393, Sigma-Aldrich; 1:200 dilution with 5\% goat serum in PBS containing 1\% BSA) for $1 \mathrm{~h}$ at room temperature, followed by 3,3'-diaminobenzidine tetrahydrochloride (Dako, Glostrup, Denmark) in the presence of $\mathrm{H}_{2} \mathrm{O}_{2}$. The slides were gently washed with deionized water and rinsed slowly, followed by dehydration through a graded series of ethanol and xylene, and mounted with DPX for light microscopy (Leica DMRXA2; Leica Microsystems, Wetzlar, Germany).

Preparation of PRP. PRP was prepared according to the method described by Nagae et al (14) with certain modifications. The procedures of PRP preparation were as follows (Fig. 1). Under general anesthesia, $10 \mathrm{ml}$ fresh blood was obtained using a syringe containing $1.0 \mathrm{ml}$ acid citrate dextrose solution $\mathrm{A}$ as anticoagulant. The whole blood was centrifuged using a centrifugation apparatus (KN70; Kubota, Tokyo, Japan) at $250 \mathrm{x}$ g for $10 \mathrm{~min}$. Subsequently, the single plasma fraction was collected and further centrifuged at $1,000 \mathrm{x} g$ for $10 \mathrm{~min}$. The precipitated platelets at the bottom of the centrifuge tube were collected with $3 \mathrm{ml}$ of the supernatant (platelet-poor plasma) to yield PRP.

Experimental grouping. ADSCs at passage 3 were utilized, and plated at $1 \times 10^{8}$ cells $/ 1$ in 12 -well culture plates containing coverslips. These wells were randomly assigned into a PRP group and a control group, each with six wells. The ADSCs in the PRP group were cultured with complete medium containing 10\% PRP. In the control group, the ADSCs were cultured with the complete medium. The culture medium was replaced every 3-4 days. 
Table I. Primer sequences of COL2A1, AGC and $\beta$-actin, product size and annealing temperature used in the reverse transcription-quantitative polymerase chain reaction analysis.

\begin{tabular}{|c|c|c|c|}
\hline Gene & Primer & $\begin{array}{l}\text { Product size } \\
\qquad(\mathrm{bp})\end{array}$ & $\begin{array}{l}\text { Annealing temperature } \\
\qquad\left({ }^{\circ} \mathrm{C}\right)\end{array}$ \\
\hline COL2A1 & $\begin{array}{l}\text { Forward: 5'-TCCTAAGGGTGCCAATGGTGA-3' } \\
\text { Reverse: 5'-AATGTCAACAATGGGAAGGGGT-3' }\end{array}$ & 112 & 61 \\
\hline AGC & $\begin{array}{l}\text { Forward: 5'-TCCGCTGGTCTGATGGACAC-3' } \\
\text { Reverse: 5'-AGGACCAACTTTGCCTTGAGGAC-3' }\end{array}$ & 101 & 55 \\
\hline$\beta$-actin & $\begin{array}{l}\text { Forward: 5'-AGATGTGGATCAGCAAGCAGGAGT-'3 } \\
\text { Reverse: 5'-TCTCGTTTCTGCGCCGTTAGGTTT-'3 }\end{array}$ & 133 & 60 \\
\hline
\end{tabular}

COL2A1, type II collagen $\alpha 1$ chain; AGC, aggrecan.

Immunofluorescence staining and toluidine blue staining. Following two weeks of culture, ADSCs in the PRP group were fixed with methanol for $10 \mathrm{~min}$, then washed with PBS for 5 min. Subsequently, PBS with Tween 20 (PBST) with $5 \%$ BSA was utilized to fix the washed cells. Goat polyclonal anti-rabbit Coll II antibody (cat. no. sc-52658; Santa Cruz Biotechnology, Inc.; 1:200 dilution in PBS) was used as a primary antibody for incubation with the induced ADSCs for $1 \mathrm{~min}$; the cells were then incubated with a fluorescein isothiocyanate (FITC) fluorescence-labeled goat anti-mouse secondary antibody (cat. no. F0257; Sigma-Aldrich; 1:200 dilution in PBS) for a further $60 \mathrm{~min}$. Following the incubation, the slides were washed with PBS and observed under a fluorescence microscope $\left(\right.$ EVOS $^{\circledR}$ FL; Life Technologies, Carlsbad, CA, USA). For toluidine blue staining, the procedures performed to fix the induced ADSCs were the same as those described for immunofluorescence staining. Then, the slides were dyed with $1 \%$ toluidine blue for $2 \mathrm{~h}$ prior to washing with PBS. Finally, the slides were mounted with neutral gum for observation.

Reverse transcription-quantitative polymerase chain reaction $(R T-q P C R)$. To compare the expression of type II collagen $\alpha 1$ chain (COL2A1) and aggrecan (AGC) mRNA expression between the PRP and control groups, RT-qPCR was performed. Following two weeks' culture, ADSCs from the PRP and control groups were harvested and subjected to RNA extraction with an RNeasy mini kit (Qiagen $\mathrm{GmbH}$, Hilden, Germany). mRNA was reverse-transcribed to complementary DNA (cDNA) using the First Strand cDNA kit (Promega Corporation, Madison, WI, USA). Then, $5 \mu 1$ of total cDNA from each sample was amplified in a final volume of $25 \mu \mathrm{l}$ of reaction mixture containing Platinum SYBR Green qPCR SuperMix-UDG ready-to-use reaction cocktail and specific primers for COL2A1, AGC and $\beta$-actin (all from Mergene; Table I). Cycling conditions were denaturation at $95^{\circ} \mathrm{C}$ for $1 \mathrm{~min}, 45$ cycles at $95^{\circ} \mathrm{C}$ for $20 \mathrm{sec}$, optimal annealing temperature (as defined in Table I) for $15 \mathrm{sec}, 72^{\circ} \mathrm{C}$ for $45 \mathrm{sec}$ and at $60-95^{\circ} \mathrm{C}$ with a heating rate of $0.1^{\circ} \mathrm{C} / \mathrm{sec}$. Target gene expression was normalized to that of $\beta$-actin. Relative gene expression was calculated with the $2^{-\Delta \Delta C t}$ formula (15).
Statistical analysis. Comparisons between groups were performed using a paired t-test. All data analysis was conducted using SPSS statistical software (version 17.0; SPSS Inc, Chicago, IL, USA). $\mathrm{P} \leq 0.01$ was considered to indicate a statistically significant result.

\section{Results}

Isolation and culture of ADSCs. It was shown in the present study that the ADSCs isolated from rabbit adipose tissue were adherent to the plastic culture flask after being cultured for $24 \mathrm{~h}$. Following four days of culture, long spindle cells were observed and the number of cells was significantly increased. Passage 3 ADSCs retained the long spindle morphology and proliferative potential.

Multi-lineage differentiation potential of ADSCs. Following the various multi-lineage induction processes, ADSCs were successfully induced toward adipogenic, osteogenic and chondrogenic lineages. After 21 days of adipogenic induction, lipid droplets were formed and confirmed by Oil Red O staining (Fig. 2A). This was not observed in the group cultured with complete medium only (Fig. 2B). The osteogenic differentiation potential of the isolated ADSCs was determined in vitro. Alizarin-red staining was positive as calcium nodules were observed after 21 days of induction (Fig. 2C). In the group cultured with complete medium only, alizarin red staining was negative (Fig. 2D). The chondrogenic differentiation potential of the ADSCs was determined in vitro by pellet culture. Following 21 days of chondrogenic induction, the diameter of the pellet was $\sim 1 \mathrm{~mm}$ (Fig. $2 \mathrm{E}$ ). The induced ADSC pellet was rich in Col II, as indicated by immunohistochemical staining (Fig. 2F).

Chondrogenic differentiation of ADSCs induced by PRP. Following two weeks of induction, ADSCs in the PRP group produced $\mathrm{Col}$ II, as indicated by immunofluorescence staining (Fig. 3A). No green fluorescent cells were seen in the control group (Fig. 3B), indicating that there was no significant expression of Col II. Toluidine blue staining indicated that PRP was a potent reagent for inducing ADSCs to produce aggrecan. Toluidine blue staining was positive in the PRP group (Fig. 3C) 

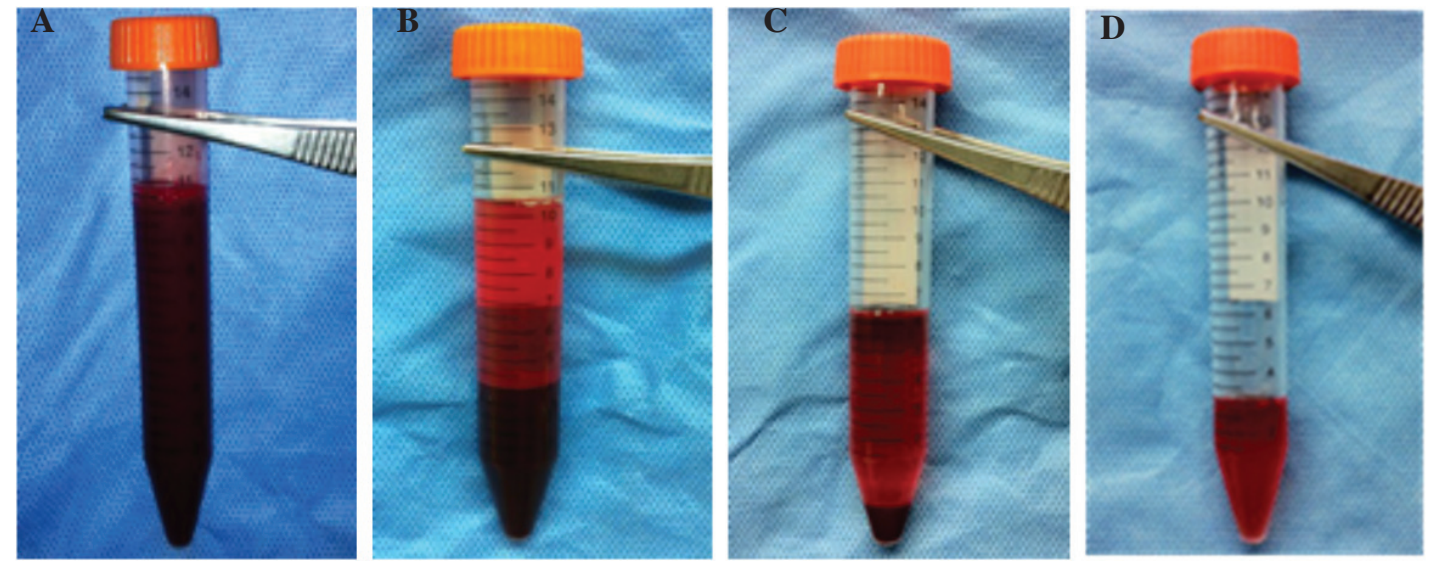

Figure 1. Preparation of autologous platelet-rich plasma (PRP). (A) A 10-ml sample of fresh blood obtained from the jugular vein of the rabbits. The sample following (B) primary centrifugation and (C) secondary centrifugation, and (D) the yield of PRP.
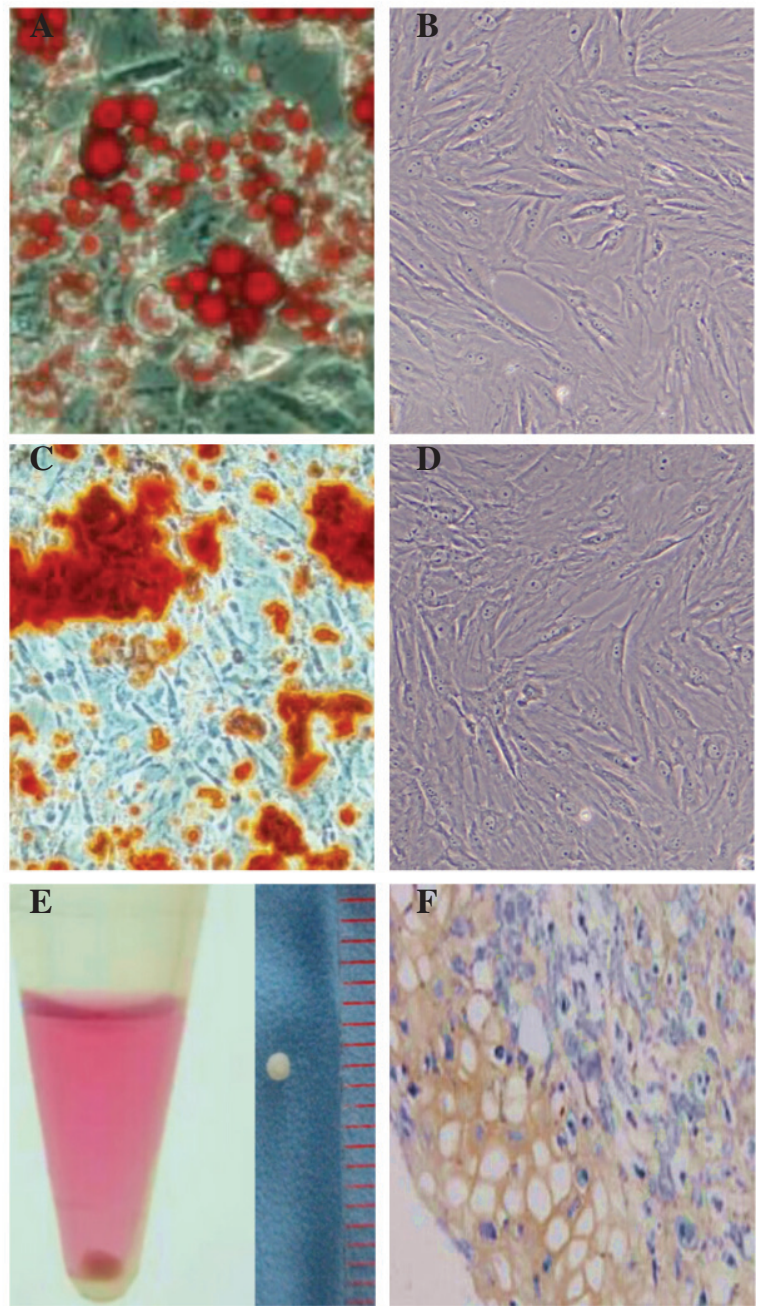

Figure 2. Cell characterization of adipose-derived stem cells (ADSCs). Oil Red O staining was (A) positive in the group cultured with adipogenic medium and (B) negative in the group cultured with complete medium. Alizarin-red staining was (C) positive in the group cultured with osteogenic medium and (D) negative in the group cultured with complete medium. (E) A cell pellet was formed in vitro. (F) Immunohistochemical staining for type II collagen was positive when the ADSC pellet was chondrogenicly induced for 21 days.

but negative in the control group (Fig. 3D). RT-qPCR confirmed that CoL2A1 and AGC mRNA expression was significantly upregulated in the PRP group compared with that in the control group $(\mathrm{P}<0.01$; Table II).

\section{Discussion}

Repair and regeneration of articular cartilage defects has been a subject of heated debate and of focused study in orthopedic 
Table II. Analysis of COL2A1 and AGC mRNA expression by ADSCs in the PRP and control groups.

\begin{tabular}{lccccr}
\hline Gene & PRP group $\Delta \mathrm{Ct}$ & Control group $\Delta \mathrm{Ct}$ & $|\Delta \Delta \mathrm{Ct}|$ & t-value & P-value \\
\hline COL2A1 & $4.28 \pm 0.06$ & $8.94 \pm 0.46$ & $4.66 \pm 0.49$ & -30.902 & 0.001 \\
AGC1 & $4.17 \pm 0.22$ & $7.30 \pm 1.43$ & $3.13 \pm 1.29$ & -12.237 & 0.047 \\
\hline
\end{tabular}

Values are presented as mean \pm standard deviation; $\mathrm{n}=6$ per group. COL2A1, type II collagen $\alpha 1$ chain; AGC, aggrecan; ADSCs, adipose-derived stem cells; PRP, platelet-rich plasma.
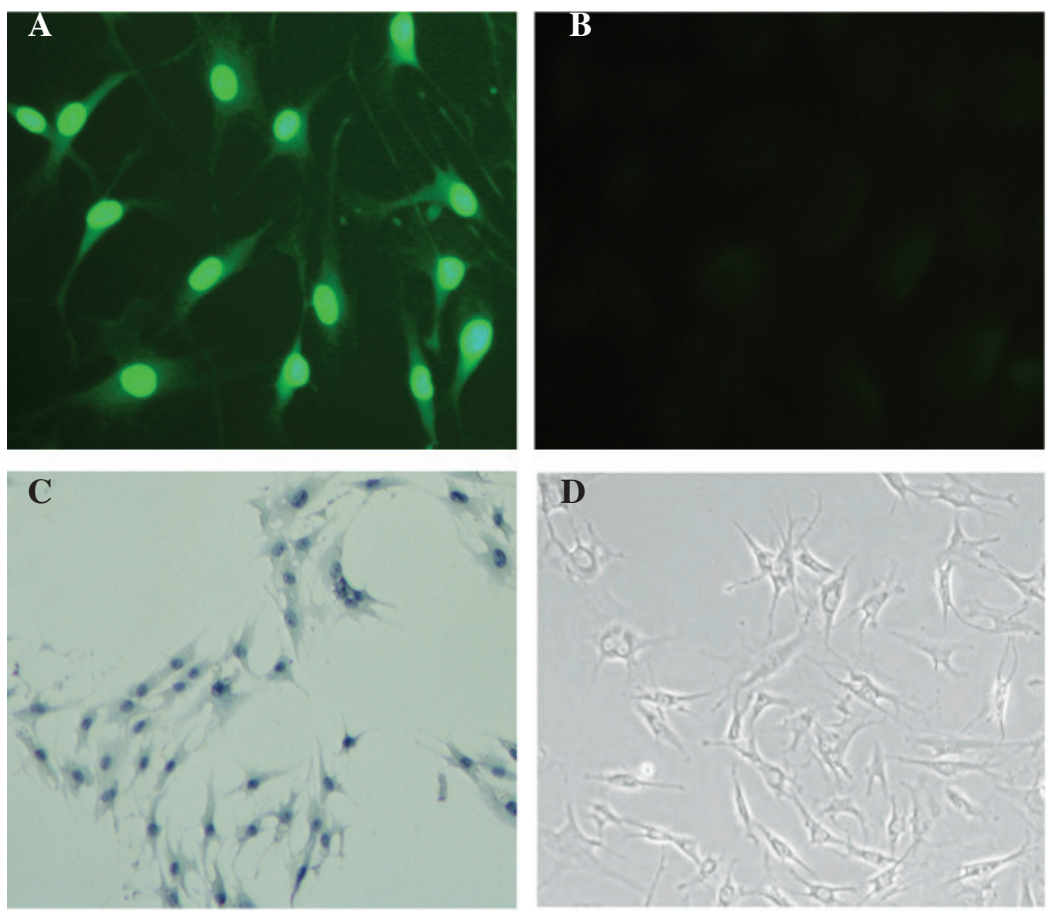

Figure 3. Type II collagen (coll II) immunofluorescence and toluidine blue-staining. Immunofluorescence staining for coll II was (A) positive in the PRP group and (B) negative in the control group. (C) Toluidine blue-staining was positive in PRP group and (D) negative in the control group. PRP, platelet-rich plasma.

research. Traditional therapeutic strategies for cartilage vary in outcome and long-term results are not satisfactory (16). In recent years, the development of tissue engineering, including bioactive growth factors, seeding cells and good bio-compatible scaffolds, has provided a wider choice for cartilage regeneration $(10,11)$. As an important factor, the choice of the seeding cells is critical for its proliferative and differentiation potential in maintaining the extracellular matrix and the function of the cartilage (17). Adult stem cells have multi-lineage differentiation potential, which could be applied in multiple tissue-engineering strategies (18). Adult stem cells are potentially useful in promoting bone healing (19), tendon injury (20), intervertebral disc degeneration (21) and cartilage injury (22), and hold promising prospects in cartilage regeneration. Among adult stem cells, bone marrow-derived stem cells (BMSCs) and ADSCs as mesoderm-derived cells have been widely used in tissue-engineering strategies for tissue regeneration. Compared with BMSCs, ADSCs are superior in the range of sources, ease of obtaining and proliferative potential, and hold a broad future in cartilage regeneration $(23,24)$.

Currently, collagenase digestion is a commonly used method to obtain ADSCs, as ADSCs have very low density in adipose tissues which mainly include Col I (25). The isolated passage 0 ADSCs in the present study became adherent to the plastic plates within $24 \mathrm{~h}$. The morphology of the passage 0 ADSCs was similar to that of fibroblasts with long spindle shapes. Then, following several passages, the ADSCs continued to maintain a fibroblastic morphology and proliferative vitality. The ADSCs were successfully isolated and their multi-lineage potential was confirmed by their induction towards adipogenic, osteogenic and chondrogenic lineages in vitro.

Finding suitable bioactive substances to induce seeding cells toward chondrogenic differentiation is a research focus of cartilage tissue engineering (26). PRP, when activated, can release multiple growth factors, including BMP-2, connective tissue growth factor (CTGF), fibroblastic growth factor-2 (FGF-2) and TGF- $\beta 2$ (5-7). BMP-2 is able to promote the proliferation and chondrogenic extracellular matrix production of adult mesenchymal stem cells (27). CTGF has the ability to stimulate mesenchymal cell proliferation, migration and aggregation (28). FGF-2 is able to upregulate proteoglycan synthesis and enhance cell proliferation $(29,30)$. TGF- $\beta 2$, as a common component of chondrogenic agent, is a potent biolog- 
ical active substance for enhancing chondrogenesis $(31,32)$. Thus, to induce ADSCs toward chondrogenic differentiation and produce cartilage related extracellular matrix, PRP was utilized. The multiple growth factors released from PRP may play a synergistic role in the differentiation of ADSCs and maintenance of cell proliferation and chondrocyte phenotype.

According to recent studies, PRP has great potential in cartilage regeneration $(33,34)$. The study by Mardani et al (33) indicated the promise of PRP, as it revealed that PRP could effectively induce human ADSCs towards chondrogenic lineage. However, currently, there is no exact definition of an ideal method of producing PRP. Different methods of isolation and activation may result in PRP of varying quality (35), for example, the presence or absence of specific factors that are essential for MSC proliferation and differentiation. In the present study, the common laboratory centrifugation method was utilized to yield PRP because it is simple and easy to perform. By reference to a study on PRP by Nagae et al (14), it was anticipated that the centrifugation steps used in that study would be helpful in the present research. Therefore, the same centrifugal force and centrifugation time were used in the present study to prepare PRP for investigation of its potency in inducing the chondrogenic differentiation of rabbit ADSCs.

In the present study, the immunofluorescence staining of Col II was positive in the PRP group but negative in the control group. Toluidine blue staining was also positive in the PRP group. The mRNA expression levels of the chondrocyte-specific markers Col2A1 and AGC were significantly upregulated. The results suggest that PRP obtained by the laboratory centrifugation procedure has potential in the chondrogenesis of ADSCs, which offers a new application in cartilage tissue engineering.

The bio-activity of PRP depends on the synergistic effects of multiple growth factors. The evaluation of the concentration of a single growth factor within PRP is not sufficient to indicate the potency of PRP. In addition to TGF- $\beta$, other growth factors exhibited in PRP, such as BMP-4, BMP-7 and GDF-5, are also important bio-activators for chondrogenic differentiation (36). In the present study, the concentrations of multiple growth factors and their synergistic effects were not tested; instead, it was anticipated that the growth factors released from PRP as a whole could be effective in inducing the chondrogenesis of rabbit ADSCs.

Laboratory centrifugation is a convenient method for obtaining PRP. However, there is no consensus on the ideal centrifugal force and time. In the present study, PRP prepared by centrifugation was confirmed to be an effective bioactive substance for inducing ADSCs towards chondrogenic lineage. Also, in this study, PRP was not activated by bovine thrombin as described in previous research (33). This was to allow the platelets in the culture medium to slowly burst and release the growth factors in a gradual way. In the future, the focus of PRP research should be to confirm the presence or absence of certain growth factors particularly associated with chondrogenesis and investigate how the synergistic effects differ according to different methods of preparation.

In summary, a common laboratory centrifugation method was used to prepare PRP, and its efficacy was confirmed when used to induce the chondrogenesis of rabbit ADSCs. The procedure used to obtain the PRP was based on that of a previous study. However, future studies are required to confirm the optimal centrifugation force and time. Also, the presence or absence of certain growth factors associated with chondrogenesis and their synergistic effects should be measured to provide further information for future clinical applications.

\section{Acknowledgements}

This study was supported by Jiangsu Health Research Program, China (No. H201258).

\section{References}

1. Lim JY, Loiselle AE, Lee JS, et al: Optimizing the osteogenic potential of adult stem cells for skeletal regeneration. J Orthop Res 29: 1627-1633, 2011.

2. Gimble JM and Guilak F: Adipose-derived adult stem cells: isolation, characterization, and differentiation potential. Cytotherapy 5: 362-369, 2003.

3. Marx RE: Platelet-rich plasma (PRP): what is PRP and what is not PRP? Implant Dent 10: 225-228, 2001.

4. Knighton DR, Hunt TK, Thakral KK and Goodson WH III: Role of platelets and fibrin in the healing sequence: an in vivo study of angiogenesis and collagen synthesis. Ann Surg 196: 379-388, 1982 .

5. Brass L: Understanding and evaluating platelet function. Hematology Am Soc Hematol Educ Program 2010: 387-396, 2010.

6. Zhang N, Wu YP, Qian SJ, et al: Research progress in the mechanism of effect of PRP in bone deficiency healing. ScientificWorld Journal 2013: 134582, 2013.

7. Lenza M, Ferraz Sde B, Viola DC, et al: Platelet-rich plasma for long bone healing. Einstein (Sao Paulo) 11: 122-127, 2013.

8. Lubkowska A, Dolegowska B and Banfi G: Growth factor content in PRP and their applicability in medicine. J Biol Regul Homeost Agents 26 (2 Suppl 1): 3S-22S, 2012.

9. Lee HR, Park KM, Joung YK, et al: Platelet-rich plasma loaded in situ-formed hydrogel enhances hyaline cartilage regeneration by CB1 upregulation. J Biomed Mater Res A 100: 3099-3107, 2012.

10. Wearing SC, Hennig EM, Byrne NM, et al: Musculoskeletal disorders associated with obesity: a biomechanical perspective. Obes Rev 7: 239-250, 2006

11. Johnstone B, Alini M, Cucchiarini M, et al: Tissue engineering for articular cartilage repair - the state of the art. Eur Cell Mater 25: 248-267, 2013.

12. Ye K, Felimban R, Moulton SE, et al: Bioengineering of articular cartilage: past, present and future. Regen Med 8: 333-349, 2013.

13. Rui YF, Lui PP, Lee YW and Chan KM: Higher BMP receptor expression and BMP-2-induced osteogenic differentiation in tendon-derived stem cells compared with bone-marrow-derived mesenchymal stem cells. Int Orthop 6: 1099-1107, 2012.

14. Nagae M, Ikeda T, Mikami Y, et al: Intervertebral disc regeneration using platelet-rich plasma and biodegradable gelatin hydrogel microspheres. Tissue Eng 13: 147-158, 2007.

15. Livak KJ and Schmittgen TD: Analysis of relative gene expression data using real-time quantitative PCR and the 2(-Delta Delta C(T)) Method. Methods 25: 402-408, 2007.

16. Kock L, van Donkelaar CC and Ito K: Tissue engineering of functional articular cartilage: the current status. Cell Tissue Res 347: 613-627, 2012.

17. Johnstone B and Yoo J: Mesenchymal cell transfer for articular cartilage repair. Expert Opin Biol Ther 1: 915-921, 2001.

18. Tollervey JR and Lunyak VV: Adult stem cells: simply a tool for regenerative medicine or an additional piece in the puzzle of human aging? Cell Cycle 10: 4173-4176, 2011.

19. Janicki P and Schmidmaier G: What should be the characteristics of the ideal bone graft substitute? Combining scaffolds with growth factors and/or stem cells. Injury 42 (Suppl 2): 77-81, 2011.

20. Lui PP, Rui YF, Ni M and Chan KM: Tenogenic differentiation of stem cells for tendon repair - what is the current evidence? J Tissue Eng Regen Med 5: e144-e163, 2011.

21. Huang S, Tam V, Cheung KM, et al: Stem cell-based approaches for intervertebral disc regeneration. Curr Stem Cell Res Ther 6: 317-326, 2011.

22. Lubis AM and Lubis VK: Adult bone marrow stem cells in cartilage therapy. Acta Med Indones 44: 62-68, 2012. 
23. Zuk PA, Zhu M, Mizuno H, et al: Multilineage cells from human adipose tissue: implications for cell-based therapies. Tissue Eng 7: 211-228, 2001.

24. Casteilla L, Planat-Benard V, Bourin P, et al: Use of adipose tissue in regenerative medicine. Transfus Clin Biol 18: 124-128, 2011 (In French).

25. Markarian CF, Frey GZ, Silveira MD, et al: Isolation of adipose-derived stem cells: a comparison among different methods. Biotechnol Lett 36: 693-702, 2014.

26. Griffin M, Hindocha S and Khan WS: Chondrogenic differentiation of adult MSCs. Curr Stem Cell Res Ther 7: 260-265, 2012

27. Freyria AM, Courtes S and Mallein-Gerin F: Differentiation of adult human mesenchymal stem cells: chondrogenic effect of BMP-2. Pathol Biol (Paris) 56: 326-333, 2008 (In French).

28. Arnott JA, Lambi AG, Mundy C, et al: The role of connective tissue growth factor $(\mathrm{CTGF} / \mathrm{CCN} 2)$ in skeletogenesis. Crit Rev Eukaryot Gene Expr 21: 43-69, 2011.

29. Ellman MB, An HS, Muddasani P and Im HJ: Biological impact of the fibroblast growth factor family on articular cartilage and intervertebral disc homeostasis. Gene 420: 82-89, 2008.

30. Stewart AA, Byron CR, Pondenis H and Stewart MC: Effect of fibroblast growth factor-2 on equine mesenchymal stem cell monolayer expansion and chondrogenesis. Am J Vet Res 68 : 941-945, 2007.
31. Van Osch GJ, Van Der Veen SW, Burger EH and Verwoerd-Verhoef HL: Chondrogenic potential of in vitro multiplied rabbit perichondrium cells cultured in alginate beads in defined medium. Tissue Eng 6: 321-330, 2000.

32. Barry F, Boynton RE, Liu B and Murphy JM: Chondrogenic differentiation of mesenchymal stem cells from bone marrow: differentiation-dependent gene expression of matrix components. Exp Cell Res 268: 189-200, 2001.

33. Mardani M, Kabiri A, Esfandiari E, et al: The effect of platelet rich plasma on chondrogenic differentiation of human adipose derived stem cells in Transwell culture. Iran J Basic Med Sci 16: 1163-1169, 2013.

34. Smyth NA, Murawski CD, Fortier LA, et al: Platelet-rich plasma in the pathologic processes of cartilage: review of basic science evidence. Arthroscopy 29: 1399-1409, 2013.

35. Anitua E, Andia I, Ardanza B, et al: Autologous platelets as a source of proteins for healing and tissue regeneration. Thromb Haemost 91: 4-15, 2004.

36. Krüger JP, Freymannx U, Vetterlein S, et al: Bioactive factors in platelet-rich plasma obtained by apheresis. Transfus Med Hemother 40: 432-440, 2013 